\title{
Identificação de problemas relacionados aos medicamentos em idosos atendidos em um laboratório de análises clínicas
}

\section{Identification of drug-related problems in the elderly attended in a clinical analysis laboratory}

\author{
Angela Maria Piva Guadagnina, Vanessa Sgnaolin ${ }^{b}$ \\ a Farmacêutica Especialista em Diagnóstico Laboratorial, Faculdade de Farmácia, Pontifícia Universidade Católica do Rio Grande do Sul, Porto Alegre, Brasil. \\ <angelampiva@yahoo.com.br> \\ b Farmacêutica, Doutoranda do Programa de Pós-Graduação em Gerontologia Biomédica, Pontifícia Universidade Católica do Rio Grande do Sul, Porto Alegre, Brasil. \\ <vanessasgnaolin@yahoo.com.br>
}

\section{ARTICLE INFO}

\section{Article history}

Received: 11/12/2014

Accepted: 17/01/2015

\section{Correspondent Author}

Vanessa Sgnaolin

Laboratório de Bioquímica, Genética

Molecular e Parasitologia

Instituto de Geriatria e Gerontologia

Pontifícia Universidade Católica do

Rio Grande do Sul

Av. Ipiranga 6690, $3^{\circ}$ andar

90610-000 Porto Alegre, RS, Brasil

<vanessasgnaolin@yahoo.com.br>

\section{(C) 2014 All rights reserved}

\section{Editors}

Alfredo Cataldo Neto

Irenio Gomes

\begin{abstract}
RESUMO
Objetivo: Quantificar e classificar os problemas relacionados aos medicamentos e avaliar a utilização dos medicamentos potencialmente inapropriados em idosos. Métodos: Estudo observacional, descritivo e transversal. Realizado em Laboratório de Análises Clínicas com indivíduos acima de 60 anos, utilizando continuamente 2 ou mais medicamentos. Os problemas relacionados aos medicamentos foram avaliados e classificados seguindo Segundo Consenso de Granada. As possíveis interações medicamentosas foram avaliadas e classificadas de acordo com a Base de Dados MICROMEDEX ${ }^{\circledR}$. A utilização de medicamentos potencialmente inapropriados para idosos foi avaliada conforme o Critério de Beers. Resultados: Os 38 idosos incluídos no estudo eram predominantemente do sexo feminino $(71,1 \%)$ e com idade média de 73,4 $\pm 9,3$ anos. Dentre as doenças crônicas relatadas as mais prevalentes foram hipertensão arterial sistêmica $(65,8 \%)$; doenças endócrinas $(65,8 \%)$, principalmente diabete mellitus $(36,8 \%)$, dislipidemia $(28,9 \%)$ e doença da tireóide $(21,0 \%)$; e transtornos mentais $(28,9 \%)$. A média de medicamentos utilizados pelos idosos foi de $6,5 \pm 2,8$. A ocorrência de polifarmácia foi $71,1 \%$. A prevalência de problemas relacionados aos medicamentos foi $86,8 \%$, com média de 3,7 e um total de 141. Os medicamentos identificados como potencialmente inapropriados para idosos foram utilizados por $44,7 \%$ dos participantes do estudo. Conclusão: Considerando que a perda da funcionalidade celular, molecular e fisiológica dos tecidos ou órgãos ocorrida no processo de envelhecimento pode impactar na absorção, distribuição e eliminação de fármacos, a população geriátrica apresenta uma grande necessidade de acompanhamento farmacológico, visando à redução dos problemas relacionados aos medicamentos e o monitoramento e/ou diminuição do uso de medicamentos potencialmente inapropriados para idosos.
\end{abstract}

DESCRITORES: Idoso. Farmacoterapia. Erros de Medicação.

\begin{abstract}
Background: To quantify and classify the drug-related problems and evaluate the use of potentially inappropriate medications in elderly. Methods: Observational, descriptive and cross-sectional study. Performed in a Clinical Laboratory with elderly over 60 years, continuously using two or more drugs. The drug-related problems were evaluated and classified following Second Consensus of Granada. Potential drug interactions were evaluated and classified according to the Micromedex ${ }^{\circledast}$ program. The use of potentially inappropriate medications for the elderly was evaluated according to the Beers Criteria. Results: The 38 elderly included in the study were predominantly female $(71.1 \%)$ and the mean age was $73.4 \pm 9.3$ years. Among the most prevalent chronic diseases reported were hypertension (65.8\%); endocrine disorders (65.8\%), especially diabetes mellitus (36.8\%), dyslipidemia (28.9\%) and thyroid disease (21.0\%); and mental disorders (28.9\%). The average number of drugs used by the elderly was $6.5 \pm 2.8$. The occurrence of polypharmacy was $71.1 \%$. The prevalence of drug-related problems was $86.8 \%$, with average of 3.7 and total 141 . The drugs identified with potentially inappropriate for the elderly were used by $44.7 \%$ of study participants. Conclusion: Considering that the loss of cellular, molecular and physiological function of tissues or organs occurred in the aging process can impact the absorption, distribution and elimination of drugs, the geriatric population presents a great need for pharmacologic follow, aiming at reducing drug-related problems and monitoring and/or decrease the use of potentially inappropriate medications for the elderly.

KEYWORDS: Aged. Drug Therapy. Medication Errors.
\end{abstract}

* Contribuição dos autores: AMP Guadagnin, elaboração e desenho do estudo, coleta de dados, banco de dados e redação do artigo; V Sgnaolin. elaboração e desenho do estudo, análise estatística, redação do artigo e revisão crítica. 


\section{INTRODUÇÃO}

O envelhecimento da população está alterando a sua distribuição etária, principalmente devido à redução das taxas de fecundidade e mortalidade, e melhoria na qualidade de vida. ${ }^{1,2} \mathrm{O}$ cuidado dos idosos demanda uma compreensão dos efeitos do envelhecimento sobre a fisiologia humana, síndromes geriátricas, incapacidades, contextos sociais e objetivos do tratamento. ${ }^{2}$ Com o envelhecimento ocorrem diversas alterações que podem interferir principalmente na farmacocinética dos medicamentos. Estas modificações compreendem: alteração da composição corporal; diminuição na secreção de saliva, enzimas digestivas, e ácido clorídrico; atrofia gástrica com diminuição do tempo de esvaziamento gástrico; diminuição da superfície de absorção e motilidade intestinal; alteração da microbiota e barreira imunológica intestinal; diminuição do tamanho e a capacidade de filtração renal e diminuição do peso, volume e fluxo sanguíneo do fígado. ${ }^{2-10}$

A presença de múltiplas doenças e das alterações fisiológicas, farmacocinéticas, farmacodinâmicas e nutricionais do envelhecimento, ${ }^{2,6,7,11}$ associadas a aumento do consumo de medicamentos, a falta de conhecimento quanto a terapia farmacológica, a elevado número de consultas médicas e a baixa frequência no uso de recursos não-farmacológicos para o manejo das doenças são alguns dos fatores de risco para o aparecimento dos Problemas Relacionados aos Medicamentos (PRMs) nos idosos. ${ }^{11}$

Os PRMs são descritos como potenciais resultados negativos decorrentes do uso de medicamentos e podem ocorrer por muitas razões, como erros de prescrição, monitorização inadequada ou fatores relacionados à forma como o paciente usa os medicamentos. ${ }^{12} \mathrm{~A}$ classificação dos PRMs é importante para a prática e pesquisa da Atenção Farmacêutica, porém um sistema de classificação universal ainda não foi adotado. ${ }^{12}$

Neste contexto, o objetivo do estudo foi quantificar e classificar os PRMs segundo o Método Dáder e, também, avaliar a utilização dos medicamentos potencialmente inapropriados em idosos, utilizando o Critério de Beers.

\section{MÉTODOS}

Estudo observacional, descritivo e transversal. Realizado em um Laboratório de Análises Clínicas na cidade de Ibiraiaras, RS, Brasil, entre Maio e Julho de 2014, com idosos acima de 60 anos em acompanhamento médico, utilizando continuamente dois ou mais medicamentos. A entrevista e o questionário de pesquisa foram realizados pela Farmacêutica Bioquímica do Laboratório, que continha informações relativas ao tratamento farmacológico, características demográficas e clínicas. Resultados laboratoriais recentes foram obtidos do prontuário do laboratório.

Os princípios ativos presentes em cada especialidade farmacêutica foram listados e classificados de acordo com o Sistema de Classificação Anatômico, Terapêutico e Químico. Os PRMs foram avaliados e classificados seguindo o Segundo Consenso de Granada sobre PRMs (2002).13 As possíveis interações medicamentosas foram avaliadas e classificadas de acordo com a Base de Dados MICROMEDEX ${ }^{\circledR}$. A utilização de medicamentos potencialmente inapropriados para os idosos foi avaliada conforme o Critério de Beers, atualizado em 2012.14

As análises estatísticas foram realizadas com o auxílio do programa SPSS (do inglês, Statistical Package for the Social Sciences), versão 20. As variáveis foram descritas por frequências, médias e desvios padrão. A prevalência de PRMs foi calculada e para comparar as frequências das diferentes variáveis entre idosos com e sem PRMs foi utilizado o Teste Qui-Quadrado de Pearson. As variáveis ordinais foram comparadas pelo Teste de Tendência Linear do Qui-Quadrado. As variáveis que, no Teste Qui-Quadrado de Pearson, apresentaram uma ou mais células com valor esperado menor do que cinco foram analisadas pelo Teste Exato de Fisher. Na comparação das variáveis dicotômicas com uma variável quantitativa foi utilizado o Teste $t$ de Student. Foram considerados significativos valores de $P<0,05$.

A pesquisa foi aprovada pela Comissão Científica da Faculdade de Farmácia e pelo Comitê de Ética em Pesquisa da Pontifícia Universidade Católica do Rio Grande do Sul, parecer número 740.497.

\section{RESULTADOS}

Os 38 idosos incluídos no estudo eram predominantemente do sexo feminino $(71,1 \%)$, da cor branca $(94,8 \%)$ e com idade média de $73,4 \pm 9,3$ anos (60-95 anos). A maioria destes idosos era casada $(55,3 \%)$, estava aposentada $(89,5 \%)$, tinha o primeiro grau completo $(52,6 \%)$ e apresentava nível salarial entre dois e cinco salários mínimos (55,3\%) (Tabela 1$)$.

$\mathrm{Na}$ Tabela 2 foram descritas as variáveis clínicas dos idosos incluídos no estudo. Observou-se que $63,9 \%$ dos participantes apresentavam anemia, 47,2\% níveis glicêmicos alterados, $67,5 \%$ índice de massa corporal classificado como sobrepeso ou obesidade, $71,4 \%$ e $77,1 \%$ pressão arterial sistólica e diastólica elevadas, respectivamente. Entretanto, a maioria 
Tabela 1. Características sociodemográficas da amostra.

\begin{tabular}{|c|c|c|}
\hline Variáveis Sociodemográficas & $\mathbf{N}$ & $\%$ \\
\hline \multicolumn{3}{|l|}{ Sexo } \\
\hline Feminino & 27 & 71,1 \\
\hline Masculino & 11 & 28,9 \\
\hline \multicolumn{3}{|l|}{ Idade } \\
\hline $60-69$ anos & 16 & 42,2 \\
\hline $70-79$ anos & 11 & 28,9 \\
\hline 80 anos ou mais & 11 & 28,9 \\
\hline \multicolumn{3}{|l|}{ Cor } \\
\hline Branca & 36 & 94,8 \\
\hline Parda/Preta & 2 & 5,2 \\
\hline \multicolumn{3}{|l|}{ Escolaridade } \\
\hline Nenhum & 2 & 5,3 \\
\hline 1-3 anos & 8 & 21,1 \\
\hline 4-7 anos & 20 & 52,6 \\
\hline 8 anos ou mais & 8 & 21,0 \\
\hline \multicolumn{3}{|l|}{ Estado civil } \\
\hline Solteiro & 6 & 15,8 \\
\hline Casado & 21 & 55,3 \\
\hline Viúvo & 10 & 26,3 \\
\hline Divorciado & 1 & 2,6 \\
\hline \multicolumn{3}{|l|}{ Trabalho } \\
\hline Não & 32 & 84,2 \\
\hline Sim & 6 & 15,8 \\
\hline \multicolumn{3}{|l|}{ Ocupação } \\
\hline Aposentado & 34 & 89,5 \\
\hline Do Lar & 2 & 5,3 \\
\hline Diarista & 1 & 2,6 \\
\hline Pensionista & 1 & 2,6 \\
\hline \multicolumn{3}{|l|}{ Renda* } \\
\hline Nenhuma & 2 & 5,3 \\
\hline $1 \mathrm{SM}$ ou menos & 15 & 39,4 \\
\hline 2 a $5 \mathrm{SM}$ & 21 & 55,3 \\
\hline
\end{tabular}

SM=Salário mínimo.

* Salário mínimo vigente na época do estudo $\mathrm{R} \$ 724,00$.

Percentuais obtidos com base no total da amostra $(n=38)$.

apresentava a avaliação do perfil lipídico (colesterol total, HDL, LDL e triglicerídeo) com valores dentro das faixas desejáveis e limítrofe. Dentre as doenças crônicas relatadas as mais prevalentes foram doenças do aparelho circulatório $(76,8 \%)$, representadas na sua maioria pela hipertensão arterial sistêmica $(65,8 \%)$; doenças endócrinas $(65,8 \%)$, principalmente diabete mellitus $(36,8 \%)$, dislipidemia $(28,9 \%)$ e doença da tireóide $(21,0 \%)$; e transtornos mentais $(28,9 \%)$, sendo a depressão $(23,7 \%)$ a doença mais referida.

Em relação à análise farmacoterapêutica, a média de medicamentos utilizados pelos idosos foi de $6,5 \pm 2,8$ (3-15 medicamentos), totalizando 246 medicamentos.
Tabela 2. Características clínicas da amostra.

\begin{tabular}{|c|c|c|}
\hline Variáveis Clínicas & $\mathbf{N}$ & $\%$ \\
\hline \multicolumn{3}{|c|}{ Hemoglobina $(\mathrm{M}>13,0 \mathrm{~g} / \mathrm{dL}$ e $\mathrm{F}>12 \mathrm{~g} / \mathrm{dL})$} \\
\hline Não adequada & 23 & 63,9 \\
\hline Adequada & 13 & 36,1 \\
\hline \multicolumn{3}{|l|}{ Glicose $(<100 \mathrm{mg} / \mathrm{dL})$} \\
\hline Não adequada & 17 & 47,2 \\
\hline Adequada & 19 & 52,8 \\
\hline \multicolumn{3}{|l|}{ Colesterol Total } \\
\hline Desejável $(<200$ mg/dL) & 17 & 60,7 \\
\hline Limítrofe (200-239 mg/dL) & 9 & 32,1 \\
\hline Alto $(\geq 240 \mathrm{mg} / \mathrm{dL})$ & 2 & 7,2 \\
\hline \multicolumn{3}{|l|}{ HDL } \\
\hline Desejável ( $\geq 60$ mg/dL) & 5 & 29,4 \\
\hline Limítrofe $(60-40 \mathrm{mg} / \mathrm{dL})$ & 9 & 52,9 \\
\hline Alto $(<40 \mathrm{mg} / \mathrm{dL})$ & 3 & 17,7 \\
\hline \multicolumn{3}{|l|}{ LDL } \\
\hline Ótimo $(<100 \mathrm{mg} / \mathrm{dL})$ & 10 & 58,8 \\
\hline Desejável (100-129 mg/dL) & 6 & 35,3 \\
\hline Limítrofe (130-159 mg/dL) & 1 & 5,9 \\
\hline Alto $(\geq 160 \mathrm{mg} / \mathrm{dL})$ & 0 & 0,0 \\
\hline \multicolumn{3}{|l|}{ Triglicerídeo } \\
\hline Desejável (<150 mg/dL) & 19 & 70,4 \\
\hline Limítrofe (150-200 mg/dL) & 4 & 14,8 \\
\hline Alto $(\geq 200 \mathrm{mg} / \mathrm{dL})$ & 4 & 14,8 \\
\hline \multicolumn{3}{|l|}{ IMC } \\
\hline Baixo peso $(<18,5)$ & 2 & 5,4 \\
\hline Normal $(18,5-24,9)$ & 10 & 27,0 \\
\hline Sobrepeso $(25,0-29,9)$ & 14 & 37,9 \\
\hline Obesidade $(>30,0)$ & 11 & 29,7 \\
\hline \multicolumn{3}{|l|}{ PAS $(<140 \mathrm{mmHg})$} \\
\hline Não adequada & 25 & 71,4 \\
\hline Adequada & 10 & 28,6 \\
\hline \multicolumn{3}{|l|}{ PAD (<90 mmHg) } \\
\hline Não adequada & 27 & 77,1 \\
\hline Adequada & 8 & 22,9 \\
\hline Doenças crônicas* & 37 & 97,4 \\
\hline Aparelho circulatório & 29 & 76,3 \\
\hline Endócrinas & 25 & 65,8 \\
\hline Transtornos Mentais & 11 & 28,9 \\
\hline $\begin{array}{l}\text { Sistema osteomuscular e do tecido } \\
\text { conjuntivo }\end{array}$ & 9 & 23,7 \\
\hline Aparelho digestivo & 8 & 21,1 \\
\hline
\end{tabular}

$\mathrm{M}=$ Masculino

$\mathrm{F}=$ Feminino.

HDL =Lipoproteínas de alta densidade (do inglês, High Density Lipoproteins).

$\mathrm{LDL}=$ Lipoproteínas de baixa densidade (do inglês, Low Density Lipoproteins).

$\mathrm{IMC}=$ Indice de Massa Corporal.

PAS $=$ Pressão Arterial Sistólica.

$\mathrm{PAD}=$ Pressão Arterial Diastólica

Percentuais obtidos com base no total de cada categoria.

* Percentuais obtidos com base no total da amostra $(n=38)$. 
Tabela 3. Classificação dos Problemas Relacionados aos Medicamentos (PRMs).

\begin{tabular}{|c|c|c|}
\hline PRMs & Descrição & Frequência $(\%)$ \\
\hline \multicolumn{3}{|c|}{ Necessidade } \\
\hline PRM 1 & Problema de saúde não tratado. & 2,1 \\
\hline PRM 2 & O paciente apresenta um problema de saúde por utilizar um medicamento que não necessita. & 2,1 \\
\hline \multicolumn{3}{|l|}{ Efetividade } \\
\hline PRM 3 & O paciente apresenta um problema de saúde por uma inefetividade não quantitativa da medicação. & 0,7 \\
\hline PRM 4 & O paciente apresenta um problema de saúde por uma inefetividade quantitativa da medicação. & 31,9 \\
\hline \multicolumn{3}{|l|}{ Segurança } \\
\hline PRM 5 & O paciente apresenta um problema de saúde por uma insegurança não quantitativa de um medicamento. & 45,4 \\
\hline PRM 6 & O paciente apresenta um problema de saúde por uma insegurança quantitativa de um medicamento. & 17,8 \\
\hline
\end{tabular}

Percentuais obtidos com base no total da categoria $(n=141)$.

Tabela 4. Comparação entre as variáveis sociodemográficas e farmacológicas dos pacientes classificados com apresentando ou não Problemas Relacionados aos Medicamentos (PRMs).

\begin{tabular}{|c|c|c|c|}
\hline Variáveis & $\begin{array}{c}\text { Sem PRMs } \\
\text { n (\%) }\end{array}$ & $\begin{array}{c}\text { Com PRMs } \\
\text { n (\%) }\end{array}$ & $\mathbf{P}$ \\
\hline \multicolumn{4}{|l|}{ Sexo } \\
\hline Feminino & $2(7,4)$ & $25(92,6)$ & \multirow{2}{*}{$0,134 *$} \\
\hline Masculino & $3(27,3)$ & $8(72,7)$ & \\
\hline Idade (média \pm DP) & $74,4 \pm 13,5$ & $73,3 \pm 8,8$ & $0,805^{+}$ \\
\hline \multicolumn{4}{|l|}{ Idade } \\
\hline 60-69 anos & $3(18,7)$ & $13(81,3)$ & \multirow{3}{*}{$0,846^{*}$} \\
\hline $70-79$ anos & $0(0,0)$ & $11(100,0)$ & \\
\hline 80 anos ou mais & $2(18,2)$ & $9(81,8)$ & \\
\hline \multicolumn{4}{|l|}{ Escolaridade } \\
\hline Nenhum & $0(0,0)$ & $2(100,0)$ & \multirow{4}{*}{$0,331 *$} \\
\hline $1-3$ anos & $2(25,0)$ & $6(75,0)$ & \\
\hline 4-7 anos & $3(15,0)$ & $17(85,0)$ & \\
\hline 8 anos ou mais & $0(0,0)$ & $8(100,0)$ & \\
\hline \multicolumn{4}{|l|}{ Renda* } \\
\hline Nenhuma & $0(0,0)$ & $2(100,0)$ & \multirow{3}{*}{$0,560 *$} \\
\hline $1 \mathrm{SM}$ ou menos & $3(20,0)$ & $12(80,0)$ & \\
\hline 2 a $5 \mathrm{SM}$ & $2(9,5)$ & $19(90,5)$ & \\
\hline \multicolumn{4}{|l|}{ Polifarmácia } \\
\hline 3-4 medicamentos & $2(18,2)$ & $9(81,8)$ & \multirow{2}{*}{$0,615^{*}$} \\
\hline 5 medicamentos ou mais & $3(11,1)$ & $24(88,9)$ & \\
\hline \multicolumn{4}{|l|}{ Morisky } \\
\hline Aderente & $2(18,2)$ & $9(81,8)$ & \multirow{2}{*}{$0,615^{*}$} \\
\hline Não aderente & $3(11,1)$ & $24(88,9)$ & \\
\hline \multicolumn{4}{|c|}{ Potenciais Interações Medicamentosas } \\
\hline Não & $4(50,0)$ & $4(50,0)$ & \multirow{2}{*}{$0,004 *$} \\
\hline $\operatorname{Sim}$ & $1(3,3)$ & $29(96,7)$ & \\
\hline \multicolumn{4}{|l|}{ Entende a prescrição médica } \\
\hline Não & $3(13,6)$ & $19(86,4)$ & \multirow{2}{*}{$1,000^{*}$} \\
\hline Sim & $2(12,5)$ & $14(87,5)$ & \\
\hline \multicolumn{4}{|c|}{ Precisa de auxílio com os medicamentos } \\
\hline Não & $4(12,9)$ & $27(87,1)$ & \multirow{2}{*}{$1,000 *$} \\
\hline Sim & $1(14,3)$ & $6(85,7)$ & \\
\hline Total & $5(13,2)$ & $33(86,8)$ & \\
\hline
\end{tabular}

$\mathrm{DP}=$ Desvio Padrão. $\mathrm{SM}=$ Salário Mínimo. $\mathrm{PRMs}=$ Problemas Relacionados aos Medicamentos.

* Teste Qui-Quadrado de Pearson.

+ Teste Qui-Quadrado
Teste $t$ de Student.
Quando considerada a média de princípios ativos utilizados esta foi de 7,1 $\pm 3,1$ (3-15 princípios ativos). A ocorrência de polifarmácia, ou seja, o uso de cinco ou mais medicamentos por um mesmo indivíduo, foi de $71,1 \%$.

A prevalência de PRMs foi $86,8 \%$ e a média 3,7 PRMs, totalizando 141 PRMs com variação de 1-15 PRMs. Em um único idoso pode ser verificado a ocorrência de 15 PRMs, sendo sete PRM 5 e quatro PRM 4 e 6. O PRM mais frequente foi o PRM 5 $(45,4 \%)$, seguido do PRM 4 (31,9\%) (Tabela 3).

A Tabela 4 apresenta uma comparação entre as variáveis sociodemográficas e farmacológicas dos idosos classificados com tendo ou não PRMs. Observou-se diferença estatística entre os grupos quando avaliada a presença de potenciais interações medicamentosas.

Os medicamentos identificados com potencialmente inapropriados para idosos segundo critério de Beers foram utilizados por $44,7 \%$ dos participantes do estudo, representando $10,2 \%$ do total de medicamentos (principalmente, estradiol oral, doxazosina e ibuprofeno). Quando separados por classe terapêutica, destacaram-se medicamentos com ação no sistema nervoso central, seguidos por medicamentos cardiovasculares.

\section{DISCUSSÃO}

O número médio de medicamentos utilizados pelos idosos foi considerado elevado. Números similares foram encontrados em estudos realizados na Áustria e Suécia. ${ }^{15,16}$ Porém, representa um valor superior ao encontrado em outras regiões do Brasil, 3,03 e 3,63 medicamentos por pessoa no Paraná e Goiás, respectivamente. ${ }^{17,18}$ A diferença no número de medicamentos usados pelos idosos pode ser justificada pelos diferentes serviços prestados à população e pelo tipo de atenção à saúde oferecida em cada região. ${ }^{18}$ 
A prevalência de polifarmácia na amostra estudada foi alta, $71,1 \%$, enquanto que a de outros estados brasileiros se mostrou inferior, entre $26,4 \%$ e $27,6 \%{ }^{17,18}$ Este dado pode estar relacionado às características da amostra estudada, já que foram inclusos somente idosos em uso de dois ou mais medicamentos.

A análise dos potenciais PRMs representa um assunto ainda controverso. Há um consenso que a morbimortalidade pode ser reduzida monitorando criteriosamente os resultados da farmacoterapia, tendo em vista que a maior parte desses problemas seria evitável. Os farmacêuticos, por sua formação e acessibilidade, podem desempenhar papel relevante nessa monitorização, colaborando com os médicos e os pacientes na garantia da efetividade e segurança dos tratamentos. ${ }^{17} \mathrm{~A}$ alta prevalência de PRMs (86,8\%) também pode estar relacionada às características da amostra estudada, embora um estudo realizado em Belo Horizonte, Brasil, tenha encontrado um número muito semelhante, $73,6 \%$, tendo como amostra a população em geral. ${ }^{19}$ Tanto o PRM 5 quanto o PRM 4, que foram os mais prevalentes, com $45,4 \%$ e $31,9 \%$, respectivamente, foram identificados principalmente por potenciais interações medicamentosas, ora aumentando a toxicidade (PRM 5 - Segurança), ora diminuindo a eficácia dos medicamentos (PRM 4 - Efetividade). Este resultado foi confirmado pela análise estatística (Tabela 4), onde foi observada uma associação entre a presença de PRMs e de potenciais interações medicamentosas $(P=0,004)$. Em um estudo realizado em Vila Velha, Brasil, a maior parte dos PRMs identificados relacionava-se com a efetividade (47,6\%), com predominância do PRM 3 (36,9\%); em seguida vieram os PRMs de segurança $(31,2 \%)$, sendo o PRM 5 o mais frequente $(28,7 \%) .20$ Outros estudos encontraram uma prevalência de potenciais interações medicamentosas de 45,8 a $65,8 \%,{ }^{16,17,21}$ considerando que estas estão diretamente ligadas a efetividade e a segurança do medicamento, possivelmente se classificadas em PRMs poderiam fornecer números similares aos encontrados em nosso estudo. A falta de uma padronização nos estudos sobre PRMs dificulta a comparação dos resultados entre os mesmos, principalmente quando confrontamos os resultados de estudos brasileiros com internacionais.

Há uma evidência crescente de questões relacionadas aos medicamentos como a prescrição inapropriada para idosos. Essa prescrição inapropriada é um importante fator de risco para reações adversas e hospitalização nos idosos. ${ }^{22,23}$ Neste estudo, os medicamentos identificados como potencialmente inapropriados para idosos, segundo critério de Beers, foram utilizados por $44,7 \%$ dos participantes, representando $10,2 \%$ do total de medicamentos, que por sua vez, quando separados por classe terapêutica, destacaram-se os medicamentos com ação no sistema nervoso central (benzodiazepínicos e antidepressivos), seguidos por medicamentos cardiovasculares. A prevalência do uso de medicamentos potencialmente inapropriados para idosos é bastante variável, sendo descrita numa faixa de 12 a $63 \%$. $^{16-18,21-24}$ Os principais medicamentos envolvidos se repetiram em outros estudos. ${ }^{18,23}$ É interessante ressaltar que os idosos são mais sensíveis aos efeitos adversos dos benzodiazepínicos, como danos cognitivos, delírio e quedas, ${ }^{14}$ e que a prescrição de medicamentos potencialmente inapropriados não necessariamente desencadeia os efeitos clínicos, porém a lista mantemse como um bom guia clínico para a revisão de medicamentos na população idosa. ${ }^{22}$ As variações notadas, se comparadas com estudos europeus, podem ser parcialmente atribuídas a diferenças nas práticas de prescrição, acesso aos medicamentos e política de cuidado ao paciente disponível nos diferentes países. ${ }^{22}$

O estudo apresenta algumas limitações metodológicas, principalmente devido ao pequeno número amostral e a seleção da amostra ter ocorrido em um único local. Os resultados obtidos podem não ser representativos da população geral, já que idosos selecionados em um laboratório de Análises Clínicas podem apresentar características diferentes daqueles oriundos da população em geral.

Este estudo concluiu que a população geriátrica, por apresentar múltiplas comorbidades, apresenta uma grande prevalência de polifarmácia e potenciais PRMs. A perda da funcionalidade celular, molecular e fisiológica dos tecidos ou órgãos ocorrida no processo de envelhecimento pode impactar na absorção, distribuição e eliminação de fármacos. Desta forma, a população geriátrica apresenta uma grande necessidade de acompanhamento farmacológico, visando à redução de PRMs e o monitoramento e/ou redução do uso de medicamentos potencialmente inapropriados para idosos.

\section{REFERÊNCIAS}

1. IBGE, Instituto Brasileiro de Geografia e Estatística. Sinopse do Censo Demográfico 2010 [citado 2014 Jan 10]. Disponível em: http://www.censo2010.ibge.gov.br/sinopse/ index.php? $\mathrm{uf}=43 \&$ dados $=26 \#$ topo piramide

2. Longo DL. Medicina Interna de Harrison. 18. ed. Porto Alegre: AMGH; 2013.

3. Salles N. Basic Mechanisms of the Aging Gastrointestinal Tract. Dig Dis. 2007; 25:112-117.

4. Aymanns C, Keller F, Maus S, et al. Review on Pharmacokinetics and Pharmacodynamics and the Aging Kidney. Clin J Am Soc Nephrol. 2010;5:314-327. 
5. Choudhury D, Levi M. Kidney aging - inevitable or preventable? Nat Rev Nephrol. 2011;7:706-717.

6. Perrie Y, Singh Badhan RK, Kirby DJ, et al. The impact of ageing on the barriers to drug delivery. J Control Release. 2012;161:389-398.

7. Britton E, McLaughlin JT. Ageing and the gut. Proc Nutr Soc. 2013; 72:173-177.

8. Rayner CK, Horowitz M. Physiology of the ageing gut. Curr Opin Clin Nutr Metab Care. 2013;16:33-38.

9. Tajiri K, Shimizu Y. Liver physiology and liver diseases in the elderly. World J Gastroenterol. 2013;19(46):8459. 8467.

10. Man AL, GichevaN, Nicoletti C. The impact of ageing on the intestinal epithelial barrier on immune system. Cell Immunol. 2014;289:112-118.

11. Ramanath KV, Nedumballi S. Assessment of MedicationRelated Problems in Geriatric Patients of a Rural Tertiary Care Hospital. J Young Pharm. 2012;4:273-278.

12. Basger BJ, Moles RJ, Chen TF. Application of drug-related problem classification systems: a review of the literature. Eur J Clin Pharmacol. 2014;70:799-815.

13. Segundo Consenso de Granada sobre Problemas Relacionados con Medicamentos. Ars Pharmaceutica. 2002;43 $(3-4): 179-187$.

14. Campanelli CM. American Geriatrics Society Updated Beers Criteria for Potentially Inappropriate Medication Use in Older Adults: The American Geriatrics Society 2012 Beers Criteria Update Expert Panel. J Am Geriatr Soc. 2012; $60(4): 616-631$

15. Bergman A, Olsson J, Carlsten A, et al. Evaluation of the quality of drug therapy among elderly patients in nursing homes. Scand J Prim Health Care. 2007;25:9-14.
16. Schuler J, Duckelmann C, Beindl W, et al. Polypharmacy and inappropriate prescribing in elderly internal-medicine patients in Austria. Wien Klin Wochenschr. 2008;120: 733-741.

17. Correr CJ, Pontarolo R, Ferreira LC, et al. Riscos de problemas relacionados com medicamentos em pacientes de uma instituição geriátrica. Rev Bras Cien Farm. 2007; 43(1):55-62.

18. Santos TRA, Lima DM, Nakatani AYK, et al. Consumo de medicamentos por idosos, Goiânia, Brasil. Rev Saúde Públ. 2013;47(1):94-103.

19. Nascimento YAN, Carvalho WS, Acurcio FAA. Drug-related problems observed in a pharmaceutical care service, Belo Horizonte, Brazil. Braz J Pharm Sci. 2009;45(2):321-330.

20. Andrade EM, Cesana ES, Ferreira NG, et al. Identificação de problemas relacionados com medicamentos nos pacientes com síndrome metabólica atendidos em uma unidade básica de saúde do município de Vila Velha - ES. Rev Ciênc Farm Básica Apl. 2007;28(3):291-299.

21. Pinto MCX. Potentially inappropriate medication use among institutionalized elderly individuals in Southeastern Brasil. Braz J Pharm Sci. 2013;49(4):709-717.

22. Chen LL, Tangiisuran B, Shafie AA, et al. Evaluation of potentially inappropriate medications among older residents of Malaysian nursing homes. Int J Clin Pharm. 2012;34: 596-603.

23. Saarelainen LK, Turner JP, Shakib S, et al. Potentially inappropriate medication use in older people with cancer: Prevalence and correlates. J Geriatr Oncol. 2014;5(4): 439-446.

24. Guaraldo L, Cano FG, Damasceno GC, et al. Inappropriate medication use among the elderly: a systematic review of administrative databases. BMC Geriatr. 2011;11:79. 